\title{
Linx
}

Revue des linguistes de l'université Paris X Nanterre

$72 \mid 2015$

Former à l'écrit universitaire, un terrain pour la linguistique?

\section{Écrits universitaires et orthographe grammaticale}

Julie Duchesne et Sophie Piron

\section{OpenEdition}

\section{Journals}

Édition électronique

URL : http://journals.openedition.org/linx/1610

DOI : 10.4000/linx.1610

ISSN : 2118-9692

Éditeur

Presses universitaires de Paris Nanterre

\section{Édition imprimée}

Date de publication : 1 septembre 2015

Pagination : 95-110

ISSN : 0246-8743

\section{Référence électronique}

Julie Duchesne et Sophie Piron, «Écrits universitaires et orthographe grammaticale », Linx [En ligne], 72 | 2015, mis en ligne le 01 mars 2016, consulté le 19 avril 2019. URL : http:// journals.openedition.org/linx/1610; DOI : 10.4000/linx.1610 


\title{
Écrits universitaires et orthographe grammaticale
}

Julie DUCHESNE et Sophie PIRON

Université du Québec à Montréal, Canada

\begin{abstract}
Résumé
Au Québec, les milieux de l'éducation, la population et les médias s'inquiètent de la piètre qualité du français écrit des étudiants en général et des futurs enseignants en particulier. Les recherches appuient ces impressions communes et montrent que les étudiants du postsecondaire éprouvent des difficultés en français écrit. Plusieurs recherches portant sur les erreurs d'étudiants universitaires en contexte de rédaction ont pointé l'orthographe grammaticale comme étant la catégorie d'erreurs la plus représentée. Toutefois, force est de constater qu'il n'y a pas de consensus en ce qui a trait à la définition de l'orthographe grammaticale. La définition que nous en proposons repose sur l' « hypothèse lexicaliste forte », telle qu'elle est utilisée en morphologie. À partir de cette définition, nous présenterons un classement des règles d'accord et nous en montrerons une application à une analyse de rédactions d'étudiants.
\end{abstract}

Mots-clés

Etudiants, rédaction, maîtrise du français écrit, orthographe grammaticale, typologie des erreurs.

La piètre qualité du français écrit des étudiants est un fait déploré dans différents pays de la francophonie. Au Québec, des recherches montrent que les étudiants québécois du postsecondaire ont des difficultés en français écrit (entre autres Asselin et McLaughlin, 1992 ; Gauvin, 2011 ; Roy et al., 1995 ; Lefrançois, 2005 ; Lefrançois et Montésinos-Gelet, 2005). En France, une recherche menée par Brissaud et Bessonnat (2001) a montré que les erreurs en orthographe grammaticale perdurent jusqu'à l'université. En Belgique, Monballin et Legros (2001) ont souligné que les insuffisances linguistiques des étudiants universitaires sont bien présentes.

Ces recherches sont difficilement comparables parce que le choix des catégories et des souscatégories de classement d'erreurs diffère sensiblement d'une étude à l'autre. De plus, la catégorie apparaissant comme la plus représentée dans les erreurs en rédaction varie, elle aussi, d'une recherche à l'autre. Il ressort cependant que l'orthographe grammaticale occupe toujours une position importante (Monballin et Legros, 2001 ; Lefrançois et al., 2008 ; Paradis et Pépin, 2010). Cette catégorie d'erreurs semble, à première vue, faire consensus, d'autant que le terme est fortement ancré dans la pratique didactique, bien qu'il ait connu d'autres formulations, en toute vraisemblance jugées équivalentes (orthographe de principe, orthographe de règle). Force est de constater pourtant que deux perspectives de description coexistent dans les études consultées au sujet de ces erreurs. D'un côté, des chercheurs englobent sous orthographe grammaticale les erreurs d'accord, de conjugaison, de genre et de nombre du nom, de la formation féminin/pluriel des noms et des adjectifs (Asselin et McLaughlin, 1992 ; Lefrançois et al., 2008). De l'autre côté, les chercheurs réduisent l'orthographe grammaticale aux erreurs d'accord et de conjugaison (Roy et al., 1995 ; Monballin et Legros, 2001 ; Paradis et Pépin, 2010). 
L'objectif que nous nous fixons dans le présent article est de réfléchir sur ce que l'on peut englober sous l'appellation d'orthographe grammaticale, et par voie de conséquence sous celle d'orthographe lexicale, en s'appuyant sur les acquis de la linguistique. Cette réflexion devrait permettre d'identifier plus clairement les difficultés que les étudiants rencontrent dans leurs écrits. Notre réflexion sur l'orthographe grammaticale débouchera sur un classement des accords que nous appliquerons à un corpus d'erreurs relevées dans des rédactions d'étudiants universitaires.

L'article se centre d'abord sur les définitions d'orthographe, d'orthographe lexicale et d'orthographe grammaticale proposées dans la littérature et expose la position adoptée ici. Il propose ensuite un classement des accords en français. Il présente enfin succinctement les résultats d'une recherche portant sur des rédactions de niveau universitaire et y applique la grille d'analyse mise au point.

\section{L'orthographe}

D'un point de vue linguistique, l'orthographe règle la façon de représenter graphiquement des phonèmes d'une langue donnée, selon une norme (Gak, 1976 ; Blanche-Benveniste et Chervel, 1978 ; Riegel et al., 1994[2009]; Cogis, 2005; Wilmet, 2007 ; Catach et al., 2008). Le fonctionnement de l'orthographe française est régi par deux principes : phonographique, le plus évident, et sémiographique (Catach, 2003 ; Catach et al., 2008). Le premier principe renvoie au rôle principal des graphèmes, qui est de représenter les phonèmes. Le second ne réfère pas aux sons, mais au sens large (grammatical et lexical) des signes écrits.

Ces deux principes fondent le plurisystème de l'orthographe française, dont les éléments de base sont les graphèmes. Ceux-ci sont classés en quatre zones (Riegel et al., 1994[2009]; Catach, 2003 ; Cogis, 2005 ; Catach et al., 2008) : les phonogrammes (phonèmes), qui relèvent du principe phonographique ; les morphogrammes (morphèmes), les logogrammes (lexèmes) et les lettres étymologiques et historiques, qui sont tous régis par le principe sémiographique.

Les graphèmes qui correspondent directement aux phonèmes sont appelés les phonogrammes. Un phonème peut correspondre à plusieurs graphèmes (par exemple, le phonème [o] correspond aux graphèmes de base $\mathrm{o}, \mathrm{au}$, eau) et inversement, un graphème à plusieurs phonèmes (c pour $[\mathrm{k}],[\mathrm{s}]$ ) (Riegel et al., 1994[2009]).

Les graphèmes chargés de transcrire les morphèmes (la plus petite unité de sens) sont des morphogrammes. Les morphogrammes sont « des marques finales écrites non prononcées (-s du pluriel, -t, -e, etc.), sauf en cas de liaison» (Riegel et al., $2009: 124$ ). Il existe deux types de morphogrammes: les morphogrammes grammaticaux, qui sont des signes de genre (ami-e), de nombre (soulier-s) et de flexion verbale (tu aim-es) (Catach et al., 1980 ; Catach, 2003 ; Catach et al., 2008), et les morphogrammes lexicaux, qui donnent des informations de séries lexicales (marques internes ou externes) (Riegel et al., 1994[2009]; Campolini et al., 1997 ; Catach, 2003 ; Cogis, 2005 ; Catach et al., 2008). Par exemple, un morphogramme lexical est la marque t dans le radical du nom chant qui relie le radical à ses dérivés chanter, chanteur.

La troisième zone de l'orthographe française est celle des logogrammes. Ce sont des notations de lexèmes, c'est-à-dire des unités significatives minimales lexicales (Martinet, 1967) (définition 
aussi retenue dans les ouvrages de Riegel et al. (2009) et de Catach et al. (2008)). "Ils constituent des "figures de mots" dans lesquelles la graphie ne fait qu'un avec le mot » (Riegel et al., 2009 : 127). En d'autres termes, il s'agit de l'ensemble des graphies qui permettent de distinguer les cas d'homophonie lexicale et grammaticale.

La quatrième zone du plurisystème orthographique regroupe les lettres étymologiques et historiques, c'est-à-dire les « lettres qui ne jouent plus aucun rôle dans le système graphique d'aujourd'hui » (Riegel et al., 2009 : 128). Les lettres étymologiques sont des témoins de la descendance du français (par rapport au latin et au grec). Par exemple, les consonnes doubles du nom ville sont des lettres étymologiques (latin villa (Le nouveau petit Robert, 2011)), purement graphiques (Riegel et al., 1994[2009]). Quant aux les lettres historiques, elles constituent des traces de l'histoire du français, telles que «la notation des anciennes voyelles nasales, par exemple, homme » (Riegel et al., 2009 : 128).

Les quatre zones $\mathrm{du}$ système orthographique $\mathrm{du}$ français (les phonogrammes, les morphogrammes, les logogrammes, et les lettres étymologiques et historiques) se répartissent inégalement dans un texte quelconque. Selon les analyses de Catach (2003) et de Catach et al. (2008), entre 80 et $85 \%$ des graphèmes sont des phonogrammes; 3 à $6 \%$ sont des morphogrammes ; 3 à $6 \%$ sont des logogrammes et 12 à $13 \%$ sont des lettres étymologiques et historiques. Ainsi, « les fondations de l'écriture sont phonogrammiques » (à plus de 80\%) (Catach et al., $2008: 27)$.

Cette description de l'orthographe, issue du domaine de la linguistique, nous servira de base pour analyser les deux types d'orthographe proposés par la pratique pédagogique, c'est-à-dire l'orthographe lexicale et l'orthographe grammaticale 2 .

\section{L'orthographe lexicale}

Les auteurs qui expliquent à quoi renvoie le terme d'orthographe lexicale tantôt en proposent une véritable définition, tantôt en présentent une caractérisation, voire exposent les phénomènes qui en font partie. Il est possible de distinguer chez ces auteurs quatre axes d'analyse, qui reposent tous sur l'orthographe des mots en tant qu'unités du lexique: l'association sons-graphies, l'association sons-graphies-sens, la façon d'écrire un mot hors contexte et l'ensemble du plurisystème de l'orthographe (excepté les morphogrammes grammaticaux).

Dans le premier axe d'analyse, les auteurs proposent que l'orthographe lexicale désigne une association sons-graphies au sein d'un mot donné. Paret (2010 : 78) explique que «l'enfant qui apprend l'orthographe lexicale [apprend] [...] l'ensemble des règles de correspondance entre des suites de phonèmes (sons) et des suites graphiques (lettres) ». Simard (1995: 145) utilise la « définition traditionnelle du mot, [c'est-à-dire] [...] l'ensemble des graphies imposées par des

\footnotetext{
${ }^{1}$ Orthographe lexicale et orthographe d'usage sont employées ici comme des synonymes. Toutefois, nous préférons l'emploi d'orthographe lexicale à orthographe d'usage, tout comme Cogis (1995) et Desnoyers (1998) : «la rencontre entre orthographe et lexique englobe davantage de faits que la traditionnelle "orthographe d'usage" » (Cogis, 1995 : 41).

${ }^{2}$ Des auteurs tels que Riegel et al. (2009) ne définissent pas l'orthographe lexicale et grammaticale.
} 
conventions linguistiques qui ne dépendent pas des règles d'accord ». En seraient donc exclus les morphogrammes grammaticaux.

Dans le deuxième axe d'analyse, l'orthographe lexicale concerne l'association sons-graphies-sens pour une unité lexicale. Blanche-Benveniste (2003 : 375) explique que «l'orthographe lexicale crée ainsi une particularisation visuelle des mots qui en assure le succès. C'est l'âme du mot, qui résiste à toutes les tentatives de réforme». Cette auteure précise que «la part lexicale de l'orthographe apporte un ensemble de significations qui en disent plus que la stricte équivalence avec la prononciation » (p. 366). Manesse et Cogis (2007 : 140) abondent dans le même sens : l'orthographe lexicale concerne « la connaissance du sens des mots et de leur emploi ».

Dans le troisième axe d'analyse, l'orthographe lexicale est appréhendée comme la façon d'écrire un mot, tel qu'il est consigné dans le dictionnaire (Cogis (2005); Grevisse et Goosse (2007[2011]) ; Lefrançois et al. (2008) ; Gauvin (2011)) : "Par orthographe lexicale, on désigne donc la part de l'orthographe qui est spécifique à chacune des unités de la langue. Les dictionnaires en recensent l'orthographe [...] » (Cogis, $2005: 41$ ). Les auteurs insistent aussi sur un fait important, qui découle de la place accordée au dictionnaire dans cette conception : l'orthographe lexicale concerne le mot hors contexte.

Enfin, dans le quatrième axe d'analyse, la caractérisation de l'orthographe lexicale est liée au plurisystème de l'orthographe française (Catach, 2003 ; Catach et al., 2008). Simard (1995) ainsi que Lefrançois et al. (2008) soulignent ainsi que l'orthographe lexicale « touche la plupart des zones du plurisystème de l'orthographe française »(Simard, 1995: 146) : les phonogrammes (transcription des sons), les morphogrammes lexicaux (-ment dans tendrement), les logogrammes ([vعR] dans un ver de terre et un verre) ainsi que les lettres étymologiques et historiques (les deux « $\mathrm{m} »$ dans le verbe commander).

Quel que soit l'axe d'analyse adopté, l'orthographe lexicale renvoie à la façon d'écrire un mot en tant qu'unité du lexique. Un certain flou persiste cependant en ce qui a trait à la transcription des morphogrammes grammaticaux, qui semblent être exclus de l'orthographe lexicale.

À l'inverse, nous proposons que l'association d'une suite de sons à une suite de graphèmes prenne place autant pour un mot dans sa forme lemmatisée, non fléchie (par exemple, l'adjectif public) que dans ses variantes flexionnelles (par exemple, publique, publics, publiques), où apparaissent divers morphèmes grammaticaux (féminin, pluriel, etc.). D'ailleurs, admettre que l'orthographe lexicale renvoie à la façon d'écrire un mot tel qu'il est consigné dans le dictionnaire, cela devrait signifier que l'on admet les différentes formes que consigne systématiquement le dictionnaire (public, publique, publics, publiques). Dans un même ordre d'idées, des homophones comme son/sont, a/à, la/là constituent des unités du lexique indépendantes qui possèdent leur propre entrée dans la nomenclature du dictionnaire. L'homophonie prend place entre des variantes flexionnelles de deux unités lexicales distinctes, ou entre une variante flexionnelle et une forme invariable : ainsi, son (soit une variante de l'unité lexicale qui est déterminant possessif, soit le nom masculin) et sont (variante de l'unité lexicale qu'est le verbe être); la (variante au féminin singulier du déterminant ou du pronom) et là (adverbe, forme invariable); a (variante du verbe être) et à (préposition, forme invariable). Ces homophones sont souvent appelés grammaticaux et intégrés au domaine de l'orthographe 
grammaticale parce que leur orthographe suppose une analyse essentiellement syntaxique permettant de déterminer la classe du mot (verbe, préposition, déterminant, etc.) et par conséquent son orthographe. Si cela est vrai, il faut ajouter que le sens peut également intervenir (la/ là, a/à, etc.). On se trouve de toute manière, pensons-nous, devant des cas d'orthographe relevant de la façon d'écrire un mot du lexique en fonction du contexte. Ces cas associent trois aspects, à savoir graphie, son, et sens, ce sont donc des cas d'orthographe lexicale. Le procédé permettant de déterminer la bonne orthographe ne doit pas présider au classement de ces cas : analyse grammaticale n'implique pas or thographe grammaticale. Des phénomènes d'accord (par exemple, sujet-verbe dans a, as) peuvent intervenir par la suite.

\section{L'orthographe grammaticale}

Il existe deux positions quant aux phénomènes rangés sous la catégorie de l'orthographe grammaticale $^{3}$. Selon la première (Chervel, 1973 ; Gak, 1976 ; Blanche-Benveniste et Chervel, 1978 ; Blanche-Benveniste, 2003 ; Guénette et al., 2004 ; Cogis, 2005 ; Grevisse et Goosse, 2007[2011]; Manesse et Cogis, 2007), l'orthographe grammaticale renvoie aux marques morphologiques (spécifiquement, les morphogrammes grammaticaux) et à l'application des règles d'accord. La définition proposée par Manesse et Cogis (2007 : 32-33) est représentative : «L'orthographe dite grammaticale concerne les marques morphosyntaxiques; elle se transmet au moyen de règles qui exigent, pour être comprises et mises en œuvre, une analyse de la langue ». Catach et al. (2008) ne fait pas usage du terme orthographe grammaticale, mais regroupe les erreurs à dominante morphogrammique (type 3). Il semble donc qu'il y ait ici établissement d'un domaine d'erreurs qui relève à la fois des morphogrammes grammaticaux et de l'application des règles d'accord.

Tableau 1. Extrait du classement des erreurs à dominante morphogrammique par Catach et al. (2008)

\begin{tabular}{|l|l|l|}
\hline \multicolumn{3}{|c|}{$\begin{array}{c}\text { 3. Erreur à dominante morphogrammique } \\
\text { 1. Les morphogrammes grammaticaux }\end{array}$} \\
\hline & Type d'erreurs & Exemples $^{5}$ \\
\hline A & $\begin{array}{l}\text { Confusion de nature, de catégorie, de genre, } \\
\text { de nombre, de forme verbale, etc. }\end{array}$ & *les journaus \\
\hline B & $\begin{array}{l}\text { Omission ou adjonction erronée d'accords } \\
\text { étroits }\end{array}$ & *les chien \\
\hline C & $\begin{array}{l}\text { Omission ou adjonction erronée d'accords } \\
\text { larges }\end{array}$ & *les travaux que les étudiants ont effectué \\
\hline
\end{tabular}

Selon la seconde position adoptée (Roy et Biron, 1991; Campolini et al., 1997; Chartrand et al., 2011 ; Gauvin, 2011), l'orthographe grammaticale fait uniquement référence à la mise en place des règles d'accord : "L'orthographe grammaticale décrit les règles d'accord des mots de classes variables dans une phrase écrite » (Chartrand et al., 2011:68); «[... Les principales règles

\footnotetext{
${ }^{3}$ Comme dit plus haut, l'orthographe grammaticale est aussi appelée orthographe de principe, orthographe de règle ou orthographe d'accord.

${ }^{4}$ Tableau légèrement modifié. Les exemples sont inspirés de ceux présentés par Catach et al. (2008).

${ }^{5}$ Les exemples sont largement inspirés de Catach et al. (2008).
} 
d'accord [sont] : les accords dans le GN, les accords régis par le sujet et les accords régis par le complément direct du verbe » (Chartrand et al., 2011 : 269). Gauvin (2011 : 57) est d'ailleurs très explicite à cet égard et établit bien la différence entre orthographes lexicale et grammaticale :

[il] importe de ne pas confondre l'orthographe grammaticale avec la morphologie flexionnelle ou grammaticale : alors que la morphologie grammaticale touche la formation du genre (-e du féminin par exemple), du nombre (-s et -x des noms et adjectifs, -nt des verbes) et de la personne (le -s de la deuxième personne du singulier), l'orthographe grammaticale concerne le transfert de ces traits dans le contexte de la grammaire de la phrase.

Nous adhérons à la seconde conception, plus étroite, pour laquelle l'orthographe grammaticale porte uniquement sur l'application des règles d'accord. Par la même occasion, nous rejetons les morphogrammes grammaticaux dans le domaine de l'orthographe lexicale. Cette affirmation requiert une certaine mise en contexte. Si la définition générale de la morphologie concentre ce domaine sur l'étude de la forme des mots (et semble entrer de plain-pied dans le domaine du lexique), il n'est pas inutile de rappeler que de nombreuses discussions ont eu et ont encore lieu quant au statut de la composante morphologique au sein du cadre général de la grammaire. Ainsi, des modèles syntaxiques optent pour 1' "hypothèse lexicaliste » (Chomsky 1970), qui considère que certains phénomènes morphologiques ne doivent pas être accomplis par le module syntaxique. Dans sa version forte (Halle, 1973 ; Lapointe, 1980 ; Lieber, 1980 ; Selkirk, 1982 ; Di Sciullo et Williams, 1987), cette hypothèse exclut de la syntaxe tous les phénomènes morphologiques et les place dans le module lexical. Cette position est assumée par de grandes théories (Lexical Functional Grammar, Generalized Phrase Structure Grammar, Head-Driven Phrase Structure Grammar et, dans une certaine mesure, le programme minimaliste) (Scalise \& Guevara 2005). Dans sa version faible (Anderson, 1982 ; Aronoff, 1976 ; Corbin, 1987 ; Scalise, 1984), l'hypothèse lexicaliste établit une distinction nette entre morphologie dérivationnelle et morphologie flexionnelle. Elle propose alors que la flexion ne soit pas réalisée dans le lexique, les affixes étant assimilés à des règles opérant ailleurs que dans le module lexical.

Si les deux versions de l'hypothèse lexicaliste ont chacune leur intérêt et leurs défauts, et que la version forte semble être actuellement en perte de vitesse, nous préférons, dans le cadre de notre réflexion sur le classement des morphèmes grammaticaux, considérer que ceux-ci sont des éléments qui ont pleinement leur place dans le lexique. Cela rejoint d'ailleurs la définition générale de la morphologie actuelle.

Ces considérations appliquées à la réflexion sur l'orthographe, il est possible d'affirmer que les morphèmes grammaticaux (morphologie flexionnelle) relèvent du lexique, et par conséquent de l'orthographe de celui-ci, c'est-à-dire de l'orthographe lexicale. L'application d'une règle de formation lexicale (spécifiquement de la morphologie flexionnelle) n'implique pas orthographe grammaticale. La répartition que nous établissons entre les erreurs d'orthographe lexicale et grammaticale repose également sur notre conception du lexique, et plus particulièrement de l'entrée lexicale d'un mot. Celle-ci se définit, entre autres mais pas uniquement, comme l'association entre un lemme (une forme abstraite, arbitraire et conventionnelle) et un paradigme flexionnel du lemme (Polguère 2008), c'est-à-dire toutes les formes que peut prendre un mot variable (nom, adjectif, etc.), un mot auquel on peut appliquer des règles de morphologie flexionnelle. Ces règles de formation des variantes flexionnelles d'un mot sont des règles qui opèrent dans le lexique. Les affixes flexionnels ne créent pas un nouveau mot, mais des formes 
différentes (graphiquement et parfois phonologiquement) d'un même mot, considéré comme une unité lexicale. Par exemple, l'adjectif MONDIAL (lemme) a pour paradigme mondial, mondiale, mondiaux, mondiales. Le lemme pointe donc souvent vers plusieurs réalisations graphiques et phonologiques qui sont associées à des traits de genre, de nombre, etc.

Il découle de cette conception du lexique que les fautes dites d'orthographe varient selon que le scripteur est en situation de transcription ou de production. Ainsi, lors d'une dictée, la situation de transcription bloque toute possibilité de forme phonologiquement erronée du paradigme (par exemple, des problèmes mondiaux ne sera pas transcrit *des problèmes mondials), à l'inverse de ce qui pourrait se produire en situation de rédaction. Dans ce cas, nous considérerions d'ailleurs que la relation d'accord est bien présente (il y a bien une marque du pluriel sur l'adjectif dans * des problèmes mondials), mais le morphème grammatical du pluriel est erroné, ce qui relève du lexique et donc de l'orthographe lexicale.

\section{L'accord}

Selon la perspective que nous adoptons, les erreurs morphologiques relèvent de l'orthographe lexicale, et l'orthographe grammaticale se limite par conséquent à l'accord en tant que mise en place d'une relation syntaxique. La définition de l'accord fait, elle, l'objet d'un consensus. Il s'agit du transfert des marques morphologiques d'un donneur (nom, pronom) à un receveur (déterminant, adjectif, verbe) (Gobbe et Tordoir, 1986 ; Grevisse et Goosse, 2007[2011] ; Riegel et al., 2009). Le transfert des marques de genre, de nombre et de personne ${ }^{6}$ repose sur une relation syntaxique établie entre deux mots.

Les règles d'accord étant relativement nombreuses, des classements ont été mis sur pied. Certains auteurs proposent qu'il y ait trois groupes de règles d'accord (Riegel et al. (2009); Chartrand et al., (1999[2011]); Boivin et Pinsonneault (2008) [les trois systèmes d'accord $\left.{ }^{7}\right]$ ). D'autres ont rassemblé les règles d'accord en deux groupes (Roy et Biron, 1991).

Les mécanismes de l'accord du français se répartissent en trois groupes pour Riegel et al. (2009: 899) : «à l'intérieur du GN [(déterminant, adjectif épithète)], dans le cadre de la phrase [(verbe, participe passé et attributs $\left.{ }^{8}\right)$ ] et au-delà des limites de la phrase (pronom représentant)] ». Ces mécanismes reposent sur l'espace syntaxique dans lequel se fait l'accord. Ils sont présentés dans le tableau 2. :

Tableau 2. Les mécanismes de l'accord par Riegel et al. (2009)

\begin{tabular}{|l|l|l}
\hline À l'intérieur du GN & Dans le cadre & Au-delà des limites
\end{tabular}

\footnotetext{
${ }^{6}$ Comme le soulignent Roy et Biron (1991), le temps et le mode ne font pas partie de cette liste, car ils ne donnent pas lieu à un accord en tant que transfert morphologique de marques de genre, de nombre ou de personne. Par exemple, dans la phrase Il faut que j'aille acheter du lait, le verbe falloir exige que le verbe aller dans la phrase subordonnée soit au mode subjonctif : il ne s'agit pas d'une règle d'accord, mais d'une dépendance syntaxique. Ducard et coll. (1995) ainsi que Brousseau et Nikiema (2001) abondent dans le même sens : les marques d'accord concernent le genre, le nombre et la personne ; le mode et le temps «viennent préciser l'interprétation modale et temporelle de l'événement que désigne le verbe » (Brousseau et Nikiema, 2001, p. 373).

${ }^{7}$ Terme employé par Boivin et Pinsonneault (2008).

${ }^{8}$ Riegel et al. (2009) ne mentionnent pas explicitement le fait que les attributs entrent dans cette catégorie, mais nous pouvons le déduire.
} 


\begin{tabular}{|l|l|l|l|}
\cline { 2 - 4 } \multicolumn{1}{c|}{} & & \multicolumn{1}{c|}{ de la phrase } & \multicolumn{1}{c|}{ de la phrase } \\
\hline \multirow{3}{*}{ Receveurs } & Déterminant & Verbe & Pronom représentant \\
\cline { 2 - 4 } & Adjectif épithète & Participe passé & - \\
\cline { 2 - 4 } & - & Attributs & - \\
\hline
\end{tabular}

Les trois systèmes d'accord proposés par Chartrand et al. (1999[2011]) ainsi que par Boivin et Pinsonneault (2008) sont différents et combinent deux critères de classement, l'espace syntaxique dans lequel l'accord prend place et les fonctions qui mènent à un accord. Dans cette perspective, les types d'accords sont l'accord dans le GN, l'accord régi par le sujet et l'accord régi par le CD. Le transfert des marques morphologiques du noyau du GN au déterminant et à l'adjectif (ainsi qu'au participe passé adjectif) constitue le premier système d'accord, soit l'accord dans le GN (terme aussi employé par Riegel et al. (1994[2009])).

Le deuxième système d'accord, soit l'accord régi par le sujet, inclut : l'accord du verbe, du participe passé employé avec être, du participe passé des verbes essentiellement pronominaux, et du noyau de l'attribut du sujet ${ }^{9}$ (Chartrand et al., 1999[2011] ; Boivin et Pinsonneault, 2008). Chartrand et al. (2011) proposent que l'accord du participe passé employé avec l'auxiliaire être dans le cas d'un verbe occasionnellement pronominal fasse partie de ce système d'accord. Même s'ils expliquent que «le pronom conjoint d'un verbe occasionnellement pronominal est complément direct du verbe, le participe passé reçoit le genre et le nombre du pronom conjoint [les garçons se sont lavés hier soir: le pronom conjoint se complément direct reprend les garçons] » (p. 274), ils classent cet accord dans l'accord régi par le sujet. Boivin et Pinsonneault (2008) classent ce type d'accord dans le troisième système d'accord.

L'accord régi par le $\mathrm{CD}$ est le troisième système d'accord : il s'agit de l'accord du noyau de l'attribut du CD, du participe passé employé avec avoir (Chartrand et al., 1999[2011]; Boivin et Pinsonneault, 2008) et du participe passé d'un verbe occasionnellement pronominal (Boivin et Pinsonneault, 2008). Il est possible de résumer les trois systèmes d'accord par le tableau 3 :

Tableau 3. Synthèse des systèmes d'accord par Chartrand et al. (1999[2011]) et Boivin et Pinsonneault (2008)

\begin{tabular}{|c|c|c|c|c|c|}
\hline \multicolumn{2}{|c|}{ L'accord dans le GN } & \multicolumn{2}{|c|}{ L'accord régi par le sujet } & \multicolumn{2}{|c|}{ L'accord régi par le CD } \\
\hline Donneur & Receveur & Donneur & Receveur & Donneur & Receveur \\
\hline \multirow{4}{*}{$\begin{array}{l}\text { Noyau du } \\
\text { GN }\end{array}$} & Déterminant & \multirow{4}{*}{$\begin{array}{l}\text { Noyau du } \\
\text { GN sujet }\end{array}$} & Verbe (ou auxiliaire) & \multirow{4}{*}{$\begin{array}{l}\text { Noyau du } \\
\text { GN } \\
\text { complément } \\
\text { direct }\end{array}$} & $\begin{array}{l}\text { Noyau du groupe } \\
\text { syntaxique } \\
\text { attribut du CD }\end{array}$ \\
\hline & Adjectif & & $\begin{array}{l}\text { Noyau du groupe } \\
\text { syntaxique attribut du } \\
\text { sujet }\end{array}$ & & $\begin{array}{l}\text { Participe passé } \\
\text { employé avec } \\
\text { avoir }\end{array}$ \\
\hline & \multirow{2}{*}{$\begin{array}{l}\text { Participe } \\
\text { passé } \\
\text { employé seul } \\
\text { (ou participe } \\
\text { passé } \\
\text { adjectif) }\end{array}$} & & $\begin{array}{l}\text { Participe passé } \\
\text { employé avec être }\end{array}$ & & $\begin{array}{l}\text { Participe passé } \\
\text { des verbes }\end{array}$ \\
\hline & & & $\begin{array}{l}\text { Participe passé des } \\
\text { verbes } \\
\text { essentiellement } \\
\text { pronominaux }\end{array}$ & & $\begin{array}{l}\text { occasionnellement } \\
\text { pronominaux }^{10}\end{array}$ \\
\hline
\end{tabular}

\footnotetext{
${ }^{9}$ Chartrand et al. (2011) mentionnent qu'il s'agit seulement du noyau adjectif du GAdj (attribut du sujet).

${ }^{10}$ Uniquement classé dans ce système d'accord par Boivin et Pinsonneault (2008).

11 Chartrand et al. (2011: 273) abordent la notion d'accord du participe passé des verbes essentiellement pronominaux dans la section «l'accord du participe passé employé avec l'auxiliaire être ».
} 


\begin{tabular}{|l|l|l|l|l|}
\hline & & $\begin{array}{l}\text { Participe passé } \\
\text { employé avec } \\
\text { l'auxiliaire être dans } \\
\text { le cas d'un verbe } \\
\text { occasionnellement }_{\text {pronominal }^{12}}\end{array}$ & & \\
\hline
\end{tabular}

Pour leur part, Roy et Biron (1991) proposent deux catégories pour les règles d'accord, qui reposent sur l'approche donneur-receveur. Ils suggèrent qu'il y ait trois types de donneurs, le sujet, l'objet et le nom, qui portent des traits de nombre, personne et genre. Les receveurs sont le verbe, l'attribut, le participe passé et les déterminés. "Un déterminé est un terme "qui est influencé formellement par...” ( Roy et Biron, 1991 : 36) : il s'agit des mots qui reçoivent les marques morphologiques du donneur nom, c'est-à-dire les déterminants et les adjectifs (Roy et Biron, 1991).

Roy et Biron (1991) ont classé les relations d'accord en deux groupes : les relations intragroupes et les relations intergroupes. Le classement repose donc sur l'espace syntaxique dans lequel l'accord prend place. Les relations intragroupes concernent le nom et le déterminé tandis que les relations intergroupes concernent les donneurs sujet et objet et les receveurs verbe, attribut et participe passé (à certaines conditions). Le tableau 4 résume le classement des règles d'accord de Roy et Biron (1991) :

Tableau 4. Les relations intra et intergroupes selon Roy et Biron (1991)

\begin{tabular}{|l|l|l|l|}
\hline \multicolumn{2}{|c|}{ Relations intragroupes } & \multicolumn{2}{c|}{ Relations intergroupes } \\
\hline \multicolumn{2}{|c|}{ Donneur } & \multicolumn{1}{c|}{ Receveur } & \multicolumn{1}{c|}{ Donneur } \\
\hline \multirow{2}{*}{ Nom } & $\begin{array}{l}\text { Déterminant } \\
\text { (simple ou complexe) }\end{array}$ & Sujet & Verbe \\
\hline \multirow{2}{*}{ Nom } & $\begin{array}{l}\text { Adjectif (devant ou } \\
\text { derrière le nom) }\end{array}$ & Sujet & Attribut \\
\cline { 3 - 4 } & & Sujet & Participe passé \\
\cline { 3 - 4 } & & Objet ${ }^{13}$ & Attribut \\
\cline { 3 - 4 } & & Objet & Participe passé \\
\hline
\end{tabular}

Les informations sur la définition des termes intergroupes et intragroupes sont cependant limitées. Il est étonnant de constater que, dans les relations intergroupes, l'accord objet-participe passé se déroule en fait à l'intérieur d'un seul groupe, le groupe verbal $(\mathrm{GV})^{14}$. En effet, dans ce cas, il s'agit de mettre en relation le noyau du groupe ayant la fonction objet (pouvant certes être déplacé en surface à l'extérieur du GV) et le participe passé, un élément du GV. Ainsi, pour Roy et Biron (1991), les relations intragroupes se déroulent spécifiquement à l'intérieur du groupe nominal (GN) alors que les relations intergroupes prennent place entre le groupe ayant la fonction de sujet et celui ayant la fonction de prédicat (receveurs verbe, attribut du sujet et participe passé) ou encore, entre des groupes à l'intérieur du GV (receveurs attribut de l'objet et participe passé).

\footnotetext{
${ }^{12}$ Uniquement classé dans ce système d'accord par Chartrand et al. (2011).

${ }^{13}$ Objet est le terme employé par Roy et Biron (1991).

${ }^{14}$ Roy et Biron souscrivent à l'existence d'un groupe verbal.
} 
Les classements passés en revue (Riegel et al. (1994 [2009]), Chartrand et al. (1999[2011]), Boivin et Pinsonneault (2008), Roy et Biron (1991)) regroupent les règles d'accord du français sur la base de deux types de critères : l'espace syntaxique (groupe, phrase et hors phrase) et les donneurs-receveurs d'accord. Riegel et al. (1994 [2009]) sont les seuls à envisager un espace syntaxique plus vaste que la phrase et, par conséquent, à considérer les pronoms représentants comme des mots qui reçoivent un accord. Le critère des donneurs-receveurs impose de traiter des classes de mots qui reçoivent des marques d'accord (nom, déterminant, adjectif, verbe et même participe passé) et des fonctions des groupes autant donneurs (sujet, complément direct) que receveurs (attribut du sujet et de l'objet). Il est à noter que Riegel et al. (1994 [2009]) ne prennent en compte que les receveurs.

Nous optons, dans le cadre de cette recherche, pour le classement général des mécanismes d'accord de Riegel et al. ([1994]2009) et pour une adaptation des classements des autres auteurs. Nous ferons usage de la notion d'accord intragroupe et intergroupe de Roy et Biron (1991), qui est en lien avec les accords étroits et larges présentés dans Catach et al. (2008).

Tableau 5. Classement des erreurs en orthographe grammaticale

\begin{tabular}{|c|c|c|c|}
\hline Sous-catégorie 1 & Sous-catégorie 2 & Sous-catégorie 3 & Exemples $^{15}$ \\
\hline \multirow{4}{*}{ Intragroupe } & \multirow{2}{*}{$\begin{array}{l}\text { Accord nom- } \\
\text { déterminant }\end{array}$} & Erreur donneur (nom) & *huit heure \\
\hline & & $\begin{array}{l}\text { Erreur receveur } \\
\text { (déterminant) }\end{array}$ & *tout les jours \\
\hline & \multirow{2}{*}{$\begin{array}{l}\text { Accord nom- } \\
\text { adjectif }\end{array}$} & Erreur donneur (nom) & *nouvelles façon \\
\hline & & Erreur receveur (adjectif) & *les points positif \\
\hline \multirow{8}{*}{ Intergroupe } & \multirow{4}{*}{$\begin{array}{l}\text { Accord régi par le } \\
\text { sujet }\end{array}$} & Accord sujet-verbe & $\begin{array}{l}\text { *Les enfants nous } \\
\text { transporte dans un } \\
\text { monde merveilleux. }\end{array}$ \\
\hline & & Accord attribut du sujet & Je suis prêtes. \\
\hline & & $\begin{array}{l}\text { Accord participe passé } \\
\text { employé avec être }\end{array}$ & $\begin{array}{l}\text { *Mes élèves ont été } \\
\text { charmé par moi. }\end{array}$ \\
\hline & & $\begin{array}{l}\text { Accord participe passé d'un } \\
\text { verbe toujours pronominal }\end{array}$ & *IIs se sont évanoui. \\
\hline & \multirow{3}{*}{$\begin{array}{l}\text { Accord régi par le } \\
\mathrm{CD}\end{array}$} & Accord attribut du CD & $\begin{array}{l}\text { *Elle les a trouvé } \\
\text { intéressant. }\end{array}$ \\
\hline & & $\begin{array}{l}\text { Accord participe passé } \\
\text { employé avec avoir }\end{array}$ & $\begin{array}{l}\text { * Ma perception a } \\
\text { changée. }\end{array}$ \\
\hline & & $\begin{array}{l}\text { Accord participe passé d'un } \\
\text { verbe occasionnellement } \\
\text { pronominal }\end{array}$ & $\begin{array}{l}\text { *Nous nous sommes } \\
\text { posés la question. }\end{array}$ \\
\hline & $\begin{array}{l}\text { Accord du } \\
\text { déterminant } \\
\text { possessif }\end{array}$ & - & $\begin{array}{l}\text { *Chaque individu est } \\
\text { unique à leur façon. }\end{array}$ \\
\hline \multirow[t]{2}{*}{ Interphrastique } & \multirow[t]{2}{*}{$\begin{array}{l}\text { Accord du } \\
\text { pronom } \\
\text { (personnel ou } \\
\text { réfléchi) }\end{array}$} & $\begin{array}{l}\text { Au sein d'une phrase } \\
\text { complexe }\end{array}$ & $\begin{array}{l}\text { *Je trouve que nous } \\
\text { sommes chanceux et } \\
\text { chanceuses de se } \\
\text { lancer... }\end{array}$ \\
\hline & & $\begin{array}{l}\text { Entre deux phrases } \\
\text { graphiques }\end{array}$ & $\begin{array}{l}\text { *Ce sont des } \\
\text { enseignants } \\
\text { passionnés. Je veux }\end{array}$ \\
\hline
\end{tabular}

${ }^{15}$ Les exemples sont tirés de notre corpus. 
Ce tableau appelle deux commentaires, le premier au sujet de l'erreur *huit heure. En grammaire moderne, l'organisation du groupe nominal autour d'un nom noyau fait du nom le donneur et du déterminant le receveur. C'est d'ailleurs ce que tous les auteurs consultés signalent. Il est dès lors préoccupant de constater que le donneur peut ne pas présenter de marque de pluriel (heure dans *huit heure, *des heure). Deux explications opposées sont envisageables.

Première explication envisagée : il faut enlever au nom son statut de noyau du groupe et le conférer au déterminant. C'est la position de la grammaire générative, en particulier depuis Abney (1987). L'hypothèse determiner phrase (DP) propose, pour des raisons indépendantes du problème d'accord soulevé ici, que le déterminant (une catégorie fonctionnelle) soit la tête du groupe. Ainsi, dans huit heures, le noyau est huit. Il n'existe donc plus de groupes nominaux en grammaire générative, mais des groupes déterminants (Radford 1997). Si l'on applique cette nouvelle analyse, le déterminant est donc le donneur et le nom, le receveur. L'erreur * huit heure s'analyse alors comme une erreur d'orthographe grammaticale dans laquelle le transfert des traits n'a pas été effectué du donneur au receveur. Nous ne suivrons pas ici l'hypothèse DP, qui ne fait pas l'unanimité en dehors de la grammaire générative et qui impose de modifier partiellement la théorie de la grammaire moderne.

Seconde explication envisagée, et c'est la position que nous adoptons : le nom conserve son statut de noyau et de donneur. Dès lors, le transfert de traits a été effectué sur le déterminant (qui présente une forme indiquant le pluriel, huit, des), mais la transcription de cette marque sur le donneur (heure), qui se place linéairement après le receveur, est « oubliée ». On se trouve devant une erreur conditionnée par la procédure d'écriture. C'est ce que Jaffré et Bessonat (1996 : 192) appellent une «erreur de gestion », et plus spécifiquement une "erreur de gestion à droite » (oubli de répéter une marque déjà apparue). Il s'agit d'une erreur liée à une surcharge cognitive. Ce type d'erreur est fréquent lorsque les transcriptions attendue et erronée ne se distinguent pas phonologiquement. Cependant, il ne serait pas impossible de le trouver dans des cas où les variantes formelles d'un lemme (JOURNAL) se distinguent phonologiquement (journal, journaux) et sont écrites en contexte de production : par exemple, *des journal ${ }^{16}$.

Ce tableau appelle un second commentaire au sujet, cette fois, des erreurs liées aux formes des déterminants possessifs (*leurs façons) et des pronoms (*de se lancer, *l'une d'elles). Rappelons que nous considérons avec Riegel et al. (2009) que les pronoms s'accordent, qu'ils sont des récepteurs de traits, tout en étant à leur tour des donneurs. Leur forme, tout comme celle des déterminants, est plus globalement dépendante du transfert de traits que ne le sont les autres mots

\footnotetext{
${ }^{16}$ À la suite de ce que nous avons dit précédemment, voici la répartition des erreurs possibles liées à l'entrée lexicale JOURNAL. L'erreur *des journal est une erreur d'accord sur le donneur, cas d'erreur de gestion à droite (oubli d'accord). *Des journals est une erreur d'orthographe lexicale : la marque du pluriel est bien présente sur le donneur (orthographe grammaticale réussie), mais le morphème grammatical du pluriel est erroné (-s). Or c'est une information contenue dans l'entrée lexicale du mot : la variante phonologique et graphique qui correspond au pluriel dans l'entrée JOURNAL n'est pas maitrisée. Cette erreur relève d'un problème d'orthographe lexicale en situation de production et explique que ce soit la composante phonologique qui est en cause. Enfin, *des journaus et *des journau présentent le même type de problème : la marque est présente sur le donneur (orthographe grammaticale réussie) puisque la phonologie du pluriel est réussie (phonème $o$ ). Cependant, les morphèmes grammaticaux, bien que réussis phonologiquement, sont erronés graphiquement (aus, au).
} 
pour lesquels l'accord se marque sur des morphèmes grammaticaux se positionnant strictement en fin de mot. Le transfert des marques a, dans ces cas-ci, un impact majeur sur les variantes morphologiques et phonologiques d'une entrée lexicale. Lorsque les variantes sont phonologiquement distinctes, la situation de production peut créer des erreurs à composante phonologique, au contraire d'une situation de transcription. Ainsi, dans la production *chaque individu est unique à leur façon, le déterminant leur présente une erreur dans le transfert des traits du possesseur, qui doit être à la $3^{\mathrm{e}}$ personne du masculin singulier (en lien avec individu). Nous considérons donc qu'il s'agit d'une erreur d'orthographe grammaticale, plus spécifiquement d'un mauvais choix dans les traits à transférer $\left(3^{e}\right.$ personne du pluriel au lieu de $3^{e}$ personne du singulier, leur au lieu de $s a$ ), ce qui a eu un impact sur le choix de la variante de l'unité lexicale du déterminant possessif.

\section{Les erreurs en orthographe grammaticale dans les rédactions d'étudiants universitaires}

Nous avons appliqué notre position théorique sur l'orthographe grammaticale à un corpus de rédactions d'étudiants. L'étude que nous avons menée a la spécificité de cibler, dans des écrits universitaires, les erreurs d'orthographe grammaticale, domaine souvent pointé par les recherches sur la maîtrise de l'orthographe (Monballin et Legros, 2001 ; Lefrançois et al., 2008 ; Paradis et Pépin, 2010). De février à avril 2011, nous avons demandé à des étudiants ${ }^{17}$ inscrits dans un baccalauréat en enseignement au Québec de rédiger un texte argumentatif d'environ 350 mots, sur un thème imposé, sans ouvrages de référence. Les étudiants touchés par notre recherche ont pour la majorité le français comme langue première $(97,62 \%)$ et un peu plus de la moitié d'entre eux sont en $3^{\mathrm{e}}$ année du baccalauréat (46/84).

Nous avons ainsi obtenu 84 rédactions dans lesquelles nous avons relevé 647 erreurs de tous genres (une moyenne de 7,70 erreurs par rédaction). Nous avons relevé plus spécifiquement 120 erreurs d'orthographe grammaticale (selon la définition étroite que nous avons adoptée), soit $19 \%$. Le tableau ci-dessous présente un aperçu des résultats et peut être utilisé comme grille de correction pour des travaux.

Tableau 6. Classement des erreurs en orthographe grammaticale

\begin{tabular}{|l|l|l|r|}
\hline \multicolumn{2}{|c|}{ Espace syntaxique (sous-catégorie 1) } & \multicolumn{2}{c|}{ Sous-catégorie 2 } \\
\hline Intragroupe & $43 \%$ & Accord nom-déterminant & $45 \%$ des intragroupes \\
\cline { 2 - 4 } & & Accord nom-adjectif & $55 \%$ des intragroupes \\
\hline \multirow{2}{*}{ Intergroupe } & $56 \%$ & Accord régi par le sujet & $83,5 \%$ des intergroupes \\
\cline { 3 - 4 } & & Accord régi par le CD & $13,5 \%$ des intergroupes \\
\cline { 3 - 4 } & & Accord du dét. possessif & $3 \%$ des intergroupes \\
\hline Interphrastique & $1 \%$ & Accord du pronom & $100 \%$ des interphrastiques \\
\hline
\end{tabular}

Les erreurs d'accord intergroupe (56\%) dominent largement les erreurs d'orthographe grammaticale de notre corpus. Au sein des erreurs d'accord intergroupe, celles régies par le sujet sont majoritaires (83,5\%). Ainsi, ce sont les erreurs d'accord en nombre et en personne du verbe, en genre et en nombre du participe passé (employé avec être) et de l'attribut du sujet qui posent surtout problème aux étudiants de notre corpus. De plus, ces erreurs relèvent de l'application

\footnotetext{
${ }^{17}$ Les sujets sont nés entre 1959 et $1991 ; 74,07 \%$ d'entre eux avaient entre 20 et 24 ans.
} 
erronée (ou de l'absence d'application) d'une règle générale d'accord et non de cas particuliers : dans l'ensemble de nos erreurs d'orthographe grammaticale, seule une (*la plupart sait... [copie 63]), peut être considérée comme une règle spécifique. À cet égard, nous arrivons à la même conclusion qu'Asselin et McLaughlin (1992:25) : ce sont les règles générales «plutôt que les irrégularités et les exceptions qui sont responsables de la majorité des erreurs [en orthographe grammaticale] [dans les rédactions] ».

\section{Conclusion}

Cet article a proposé une réflexion sur les concepts d'orthographes lexicale et grammaticale. Les propositions avancées sur la restriction de l'orthographe grammaticale à des faits strictement liés au transfert de traits lors de l'accord rejoignent celles faites antérieurement par d'autres chercheurs. Cette position est cependant moins représentée dans la littérature. Par ailleurs, notre position sur l'orthographe lexicale, la répartition entre orthographe lexicale et orthographe grammaticale, les justifications que nous avons proposées et les exemples que nous avons tenté d'analyser finement montrent à quel point le problème est complexe, requiert une analyse linguistique et engage une certaine vision du lexique, de la morphologie et de la syntaxe. Nos propositions se détachent vraisemblablement de la direction généralement adoptée face à ce genre de question, mais nous pensons qu'elles permettent de distinguer plus finement certaines erreurs rencontrées dans des rédactions d'étudiants.

Dans la partie appliquée de cet article, nous avons proposé une étude qui a permis de constater que l'orthographe grammaticale représente une part importante d'erreurs dans les écrits universitaires (environ $20 \%$ des erreurs), et ce, même en adoptant une définition étroite (limitée aux relations d'accord.

Notre recherche présente évidemment certaines limites. Le simple fait d'avoir choisi la rédaction comme instrument de collecte de données a certainement orienté nos résultats. En effet, les étudiants ont, selon toute vraisemblance, utilisé des stratégies d'évitement. C'est probablement pour cette raison que les erreurs rencontrées relèvent essentiellement des règles générales d'accord. De plus, nous ne pouvons expliquer les causes des erreurs d'orthographe grammaticale : seule une entrevue avec les étudiants nous aurait permis de mieux comprendre leurs erreurs.

Nous espérons dans tous les cas que les réflexions menées ici et les conclusions de notre recherche permettront de mieux cibler les notions des cours de français à l'université et la façon dont celles-ci seront abordées.

\section{Bibliographie}

ABNEY, S. P., 1987, The English Noun Phrase in its Sentential Aspect, Ph.D. dissertation, MIT. ANDERSON, S. R., 1982, “Where's Morphology?”, Linguistic Inquiry, 13, p. 571-612.

ARONOFF, M., 1976, Word Formation in Generative Grammar, Cambridge/London, MIT Press. 
ASSELIN, C., MC LAUGHLIN, A., 1992, «Les erreurs linguistiques rencontrées dans les écrits des étudiants universitaires : analyse et conséquences », Revue de l'Association canadienne de linguistique appliquée, 14-1, p. 13-30.

BLANCHE-BENVENISTE, C., 2003, «L'orthographe », dans M. YAGUELLO (dir.), Le grand livre de la langue française, Paris, Seuil, p. 245-389.

BLANCHE-BENVENISTE, C., CHERVEL, A., 1978, L'orthographe, Paris, F. Maspero.

BOIVIN, M.-C., PINSONNEAULT, R., 2008, La grammaire moderne : description et éléments pour sa didactique, Montréal, Éditions Chenelière Éducation.

BRISSAUD, C., BESSONNAT, D., 2001, L'orthographe au collège : pour une autre approche, Grenoble-Paris, CRDP de l'Académie de Grenoble-Delagrave.

BROUSSEAU, A.-M., NIKIEMA, E., 2001, Phonologie et morphologie du français, Montréal, Fides.

CAMPOLINI, C., VAN HÖVELL, V., VANSTEELANDT, A., 1997, Dictionnaire de la logopédie, Volume 3, Louvain-la-Neuve, Peeters.

CATACH, N., 2003, L'orthographe, Paris, Presses universitaires de France.

CATACH, N., DUPREZ, D., LEGRIS, M., 1980, L'enseignement de l'orthographe, Paris,

Fernand Nathan.

CATACH, N., GRUAZ, C., DUPREZ, D., 2008, L'orthographe française. Traité théorique et pratique avec des travaux d'application et leurs corrigés, Paris, Armand Colin.

CHARTRAND, S.-G., AUBIN, A., BLAIN, R., SIMARD, C., 1999, Grammaire pédagogique du français d'aujourd'hui, Montréal, Graficor.

CHARTRAND, S.-G., AUBIN, A., BLAIN, R., SIMARD, C., 2011, Grammaire pédagogique du français d'aujourd'hui, Montréal, Chenelière Éducation.

CHERVEL, A., 1973, «La grammaire traditionnelle et l'orthographe », Langue française, 20, p. 86-96.

CHOMSKY, N., 1970, "Remarks on nominalizations", dans R. JACOBS, P. ROSENBAUM (dir.), Readings in Transformational Grammar, Boston, Ginn, p. 111-176.

COGIS, D., 2005, Pour enseigner et apprendre l'orthographe, Paris, Delagrave.

CORBIN, D., 1987, Morphologie dérivationnelle et structuration du lexique, Paris, Niemeyer.

DESNOYERS, A., 1998, «L'orthographe lexicale, une question d'organisation des données à mémoriser », Correspondance 4-1, <http://www.ccdmd.qc.ca/correspo/Corr4-1/Desnoy.html> DI SCIULLO, A.-M., WILLIAMS, E., 1987, On the Definition of Word, Cambridge, MIT Press. DUCARD, D., HONVAULT, R., JAFFRÉ, J.-P., 1995, L'orthographe en trois dimensions, Paris, Nathan.

GAK, V.G., 1976, L'orthographe du français. Essai de description théorique et pratique, Paris, Société d'études linguistiques et anthropologiques de France (SELAF).

GAUVIN, I., 2011, Interactions didactiques en classe de français : enseignement/apprentissage de l'accord du verbe en première secondaire, Thèse de doctorat, Université de Montréal, Canada. GOBBE, R., TORDOIR, M., 1986, Grammaire française, Bruxelles, Éditions du Trécarré.

GREVISSE, M., GOOSSE, A., 2007, Le bon usage, Bruxelles, Éditions De Boeck.

GREVISSE, M., GOOSSE, A., 2011, Le bon usage, Bruxelles, Éditions De Boeck.

GUÉNETTE, L., LÉPINE, F., ROY, R.L., 2004, Le français tout compris : guide d'autocorrection du français écrit, Saint-Laurent, Éditions du Renouveau pédagogique.

HALLE, M. 1973, «Prolegomena to a theory of Word formation », Linguistic Inquiry, 4, p. 3-16. JAFFRÉ, J.-P., BESSONNAT, D., 1996, «Gestion et acquisition de l'accord: erreurs et étiologie », Faits de langue, $8: 185-192$.

LAPOINTE, S., 1980, A theory of Grammatical Agreement, Thèse de doctorat, University of 
Massachusetts, États-Unis.

Le nouveau petit Robert: dictionnaire analogique et alphabétique de la langue française (version électronique), 2011.

LEFRANCOIS, P., 2005, "How do university students solve linguistic problems? A description of the processes leading to errors", L1-Educational Studies in Language and Literature, 5-3, p. 417432.

LEFRANCOIS, P., LAURIER, M., LAZURE, R., CLAING, R., 2008, Évaluation de l'efficacité des mesures visant l'amélioration du français écrit du primaire à l'université, Montréal (Québec), Office québécois de la langue française.

LEFRANCOIS, P., MONTESINOS-GELET, I., 2005, «Les futurs enseignants et la didactique du français écrit : l'impact de la compétence perçue et réelle sur l'évolution des représentations », Revue canadienne d'enseignement supérieur, 35-2, p. 1-26.

LIEBER, R., 1980, On the Organization of the Lexicon, Thèse de doctorat, University of Massachusetts, États-Unis.

MANESSE, D., COGIS, D., 2007, Orthographe : à qui la faute, Issy-les-Moulineaux, ESF éditeur.

MARTINET, A., 1967, Éléments de linguistique générale, Paris, Armand Colin.

MONBALLIN, M., LEGROS, G., 2001, «La maîtrise langagière à l'entrée des études universitaires: mythes, constats et essais d'intervention », Correspondance, 6-4, $<$ http://www.ccdmd.qc.ca/correspo/Corr6-4/Mythes.html $>$.

PARADIS, P., PÉPIN, L., 2010, «Un outil de diagnostic basé sur les lacunes en français écrit d'étudiants en formation des maîtres », Communication présentée au colloque annuel du RUSAF, $<$ http://www.fse.uqam.ca $>$.

PARET, M.-C., 2010, « Le système de l'orthographe française », Québec français, 158, 78-80.

POLGUĖE, A., 2008 [2003], Lexicologie et sémantique lexicale. Notions fondamentales, Nouvelle édition revue et augmentée, Montréal, Presses de l’Université de Montréal.

RADFORD, A., 1997, Syntax : A minimalist introduction, Cambridge, Cambridge University Press.

RIEGEL, M., PELLAT, J.-C., RIOUL, R., 1994, Grammaire méthodique du français, Paris, Presses Universitaires de France.

RIEGEL, M., PELlAT, J.-C., RIOUL, R., 2009, Grammaire méthodique du français, Paris, Presses Universitaires de France.

ROY, G.-R., BIRON, H., 1991, S'approprier l'orthographe grammaticale par l'approche « donneur-receveur », Sherbrooke, Éditions du CRP.

ROY, G.-R., LAFONTAINE, L., LEGROS, C., 1995, Le savoir grammatical après treize ans de formation, Sherbrooke, Éditions du CRP.

SCALISE, S., 1984, Generative Morphology, Dordrecht, Foris.

SCALISE, S., GUEVARA, E., 2005, «The Lexicalist Approach to Word-Formation and the Notion of the Lexicon », dans P. ŠTEKAUER, R. LIEBER (dir.) Handbook of Word-Formation. Dordrecht, Kluwer, p. 147-187.

SIMARD, C., 1995, «L'orthographe d'usage chez les étudiants des ordres postsecondaires », Revue des sciences de l'Éducation, 21-1, p. 145-165.

WILMET, M., 2007, Grammaire rénovée du français, Bruxelles, De Boeck. 